\title{
COVID-19: Simulation Study of Tocilizumab and Siltuximab Interventions in the Context of Acute Respiratory Distress Syndrome
}

\author{
Eileen Doyle ${ }^{1}$, Darren Bentley², and Michael Dodds ${ }^{1}$ \\ ${ }^{1}$ Certara LP \\ ${ }^{2}$ Affiliation not available
}

June 11, 2020

\begin{abstract}
Aim: To assess the potential of interleukin-6 (IL-6) signaling blockade in the lung to treat SARS-CoV-2 infection via modelbased simulation by exploring soluble IL-6 receptor (sIL-6R) sequestration by tocilizumab (TCZ) and IL-6 sequestration by siltuximab (SIL). Methods: Literature values of IL-6, the IL-6 antagonist SIL, sIL-6R, the IL-6R antagonist TCZ, and their respective binding constants were used to develop a model to predict the impact of treatment on IL-6 signaling. Models were used to generate simulated bronchoalveolar lavage (BAL) concentrations for normal subjects, subjects at risk of developing acute respiratory distress syndrome (ARDS), and subjects with ARDS were simulated under four conditions: without treatment, treatment with TCZ, treatment with SIL, and treatment with TCZ + SIL. Results: With TCZ intervention, IL-6 levels are unaffected and sIL-6R is reduced somewhat below the Normal case. IL-6:sIL-6R complex only slightly decreased relative to the no-intervention case. With SIL intervention, sIL-6R levels are unaffected and IL-6 is greatly reduced below the Normal case. IL-6:sIL-6R complex is greatly decreased relative to the no-intervention case. With TCZ + SIL intervention, IL-6 and sIL-6R levels are reduced below the Normal case and achieve suppression equivalent to monotherapy results for their respective targets. IL-6:sIL-6R complex reduction is predicted to be greater than monotherapy. This reflects sequestration of both components of the complex and the nonlinear binding equilibrium. Conclusion: Co-administration of both IL-6 and IL-6R sequestering products such as SIL and TCZ may be necessary to effectively treat COVID-19 patients who have or are at risk of developing ARDS.
\end{abstract}

\section{Introduction}

To date, the most serious symptoms from COVID-19 are pulmonary complications. An overwhelming number of patients with COVID-19 present with pneumonia and acute respiratory failure. Acute respiratory distress syndrome (ARDS) involves accumulation of pro- and anti-inflammatory cytokines in the lung parenchyma that is associated with severe injury to lung epithelium and endothelium [1]. Common risk factors for the development of ARDS relevant to COVID-19 infection include pneumonia, pulmonary vasculitis, and nonpulmonary sepsis [2]. Progression to ARDS is characterized by chest imaging (positive for bilateral opacities not fully explained by pleural effusions), respiratory failure not fully explained by cardiac failure or fluid overload, and oxygen requirement. In the Berlin definition [2], ARDS is characterized as mild, moderate, or severe according to the extent of hypoxemia.

Cytokine expression shifts in patients with ARDS and those at risk for ARDS. Changes in interleukin-6 (IL-6) and IL-8, for example, have been used to predict clinical outcome (mortality) in ARDS patients [3]. IL-6 plays multiple roles in the immune response. IL-6 signaling mediates effects in the vascular wall 
such as vascular permeability, activation of the endothelium, recruitment of immune cells, and endothelial dysfunction. The result is often vascular hypertrophy and fibrosis [4]. In one study, individual cytokines increased in patients before and after the onset of ARDS, yet greater increases occurred in cognate receptors and/or antagonists, so that the molar ratios of agonists to antagonists declined dramatically at the onset of ARDS and remained low for at least 7 days. In this study, IL-6 increased an average 400-fold (mean peak $1230 \mathrm{pg} \mathrm{mL}-1$ ) over normal in 53 patients on the first day of ARDS, while sIL-6R increased only 2-3 fold. IL-6 steadily declined in these patients from Day 1-21 of ARDS, but remained elevated compared with normal levels. Importantly, the molar ratios of IL-6 and its cognate receptor sIL-6R (a specific agonist) increased more than 10-fold in patients at risk for ARDS and approximately 100-fold in patients with ARDS [5]. In another study, plasma IL-6 levels $>400 \mathrm{pg}$ mL-1 on any day in the first week of ARDS was associated with a low likelihood of survival [6]. In a third study, serum IL-6 and IL-8 levels on day 1 were significantly higher in all the ARDS patients as compared to healthy controls. As compared to survivors, the IL-6 and IL-8 levels were significantly higher in non-survivors measured on day 1. By using acute physiology and chronic health evaluation (APACHE) II score, IL-6, and IL-8, the receiver operating characteristic curve was plotted, and the provided predictable accuracy of mortality (outcome) was $94 \%$ [3].

IL-6 pathway blockade has been used to successfully treat rheumatoid arthritis and Castleman's disease [7, $8]$ and to prevent cytokine release syndrome (CRS) arising from chimeric antigen receptor T-cell therapy [9, 10]. The rationale for exploring drugs that target the IL-6 pathway in the treatment of COVID-19 is based on their utility in managing CRS. Clinically, both TCZ (which binds the IL-6 receptor and is approved by the US Food and Drug Administration (FDA) and the European Medicines Agency (EMA) for management of CRS) and SIL (which binds IL-6) are used to control CRS [9].

In the context of the COVID-19 pandemic, several countries have issued emergency use guidelines for TCZ in the treatment of severe COVID-19 pneumonia, and EUSA Pharma has instituted a compassionate use program with SIL to treat patients with COVID-19 who have developed serious respiratory complications. Additionally, there are 48 studies of TCZ and 3 studies investigating SIL for COVID-19 treatment as of June 1, 2020 (clinicaltrials.gov).

\section{Siltuximab Clinical Experience}

SIL (SYLVANT ${ }^{\circledR}$ ) is an IL-6 antagonist indicated for the treatment of patients with multicentric Castleman's disease who are HIV negative and HHV-8 negative, approved in 2014 by the FDA. SIL is administered as an $11 \mathrm{mg} \mathrm{kg}-1$ IV dose given over 1 hour every 3 weeks. SIL is not approved for the treatment of CRS, but has been administered as a single intravenous (IV) dose of $11 \mathrm{mg} \mathrm{kg}-1$ IV for this indication. Researchers in Belgium will compare the time to clinical improvement in adult patients with acute hypoxic respiratory failure and systemic CRS receiving SYLVANT $11 \mathrm{mg}$ kg-1 IV (COV-AID trial; NCT04330638; Treatment of COVID-19 Patients with Anti-interleukin Drugs) versus other therapies (standard of care, anakinra, TCZ, TCZ + anakinra, SIL + anakinra). Primary completion of the trial is estimated as September 2020, with study completion estimated December 2020.

\section{Tocilizumab Clinical Experience}

TCZ (ACTEMRA ${ }^{\circledR}$ ) is an IL-6 receptor inhibitor with several indications (rheumatoid arthritis, giant cell arteritis, polyarticular juvenile idiopathic arthritis, systemic juvenile idiopathic arthritis, cytokine release syndrome), approved in 2018 by the FDA. For the management of CRS, the recommended dose is $8 \mathrm{mg} \mathrm{kg-1}$ IV over 1 hour for patients at or above $30 \mathrm{~kg}$ and $12 \mathrm{mg} \mathrm{kg}-1$ for patients less than $30 \mathrm{~kg}$ in weight. Doses exceeding $800 \mathrm{mg}$ per infusion are not recommended in CRS patients. In the COV-AID trial (NCT04330638), TCZ will be administered as a single IV infusion at a dose of $8 \mathrm{mg} \mathrm{kg}-1$ with a maximum infusion of $800 \mathrm{mg}$ per injection. 


\section{Background}

IL-6 is critical for B-cell differentiation and maturation with secretion of immunoglobulins, cytotoxic T cell differentiation, macrophage and monocyte function and production of acute phase proteins [11]. IL-6 has both a pro- and anti-inflammatory effects [5], but its pro-inflammatory mechanisms dominate in the context of CRS and ARDS [11]. IL-6 is elevated in plasma and bronchoalveolar lavage fluid (BALF) and is predictive of poor outcomes in acute lung injury patients $[6,12]$. Higher molar ratio of IL-6:sIL-6R is associated with higher risk of death $[3,5]$. A significant anti-inflammatory response early in ARDS may provide a key mechanism for limiting the net inflammatory response in the lungs [5].

In contrast to other receptors and antagonists (such as tumor necrosis factor alpha (TNF- $\alpha$ ) and interleukin-1 (IL-1)), the molar ratio of IL-6 to sIL-6R increased more than 10-fold in BALF from patients at risk for ARDS, and was approximately 100-fold higher than in normal subjects [5].

IL-6 signaling is achieved through two signaling pathways: classic and trans [Figure 1]. In classic IL-6 signaling, IL-6 first binds membrane-bound IL-6R, then the complex binds with and dimerizes the glycoprotein 130 (gp130) transmembrane protein to elicit signal transduction [13]. The transmembrane protein IL-6R is only found on certain cell types (such as macrophages, neutrophils, some T cells, and all hepatocytes).

IL-6 carries out its signaling in other cell types via a second signaling pathway: trans signaling. This alternative activation relies on the soluble IL-6R (sIL-6R), which is constitutively present in all tissue types at ng mL-1 concentrations [13]. In trans signaling, IL-6 binds sIL-6R and the complex has the ability to bind and dimerize gp130, leading to signaling. Activation of gp130 via trans-signaling is crucial for lymphocyte trafficking into the inflamed area by controlling chemokine expression $[14,15]$. IL-6 trans-signaling promotes T-cell proliferation (for example, during colon cancer development) and is involved in regulating adhesion molecule expression on endothelial cells $[16,17]$.

One additional player in trans signaling is soluble gp130 (sgp130), which is also constitutively present [18, 19, 20]. sgp130 binds the IL-6:sIL-6R complex with high affinity ( $10 \mathrm{pM})$. Since sgp130 can bind to the IL-6:sIL-6R complex in the circulation, it acts as a specific inhibitor of IL-6 mediated trans signaling [21].

Taken together, sgp130 and sIL-6R serve to buffer levels of circulating IL-6 [22].

\section{Figure 1}

The complexity of IL-6 signaling gives insight into why blockade of IL-6 (via, for example, SIL) or IL-6R (via, for example, TCZ) alone may not be sufficient to decrease IL-6-mediated signal transduction to a clinically relevant extent in COVID-19-related ARDS.

\section{Methods}

\section{Monoclonal Antibody Systemic Concentration}

The mean peak serum concentration of SIL following the first dose of SIL $11 \mathrm{mg} \mathrm{kg-1}$ IV in patients with multicentric Castleman's disease was derived from the mean steady-state maximum concentration (Cmax) of $322 \mu \mathrm{g} \mathrm{mL}-1$ divided by the accumulation ratio (1.7), or $195 \mu \mathrm{g} \mathrm{mL}-1$ [23]. A dissociation equilibrium binding constant (Kd) for SIL has been reported as $15 \mathrm{pM}$ [24].

The mean peak serum concentration of TCZ following the first dose of TCZ $8 \mathrm{mg} \mathrm{kg}-1$ in patients with cytokine release syndrome (CRS) during chimeric antigen receptor T-cell (CAR-T) treatment is $99.5 \mu \mathrm{g}$ mL-1 [25]. A Kd for TCZ has been reported as 1240 pM [26]. 


\section{Monoclonal Antibody Concentration in the Lung}

Biodistribution mechanisms and data for therapeutic monoclonal antibodies were taken from published literature [27]. Specifically, distribution to the lung has been studied in the context of respiratory syncytial virus (RSV), where anti-RSV antibodies have been developed. BALF concentrations have been quantified in studies in cynomolgus monkeys relative to serum concentrations, and suggest extremely low BALF:Serum concentration ratios of $0.1-0.2 \%$.

For the purpose of these calculations, an optimistic BALF:Serum concentration ratio of $0.2 \%$ was used. Applying this ratio to peak SIL and TCZ serum concentrations, peak BALF concentrations are then 2690 $\mathrm{pM}$ and $1370 \mathrm{pM}$, respectively.

\section{IL-6 and sIL-6R Concentration in the Lung}

Measurements of IL-6 and sIL-6R concentrations in normal subjects, patients at-risk for ARDS, and subjects experiencing ARDS were obtained from the literature [5]. In this study, patients with sepsis or trauma who were at risk for ARDS and patients with ARDS (all cause) were identified by prospective screening of all patients admitted to the Medical and Surgical intensive care unit in a three-year period. These patients would correspond to patients on the World Health Organization Ordinal Scale for Clinical Improvement of 3 (hospitalized with mild disease, no oxygen requirement) to 7 (hospitalized with severe disease, ventilated plus additional organ support) [R\&D Blueprint COVID-19 Therapeutic Trial Synopsis Draft Feb 18, 2020]. Vital signs, including physiologic parameters of hypoxemia (expressed as partial pressure of oxygen $(\mathrm{PaO} 2)$ to fraction of expired oxygen (FIO2) ratio, tidal volume, and static lung compliance), were measured daily but not reported. Patients were not stratified by mild, moderate or severe ARDS, and the oxygen requirements of individual patients were not reported.

For the purpose of the present analysis, with respect to IL-6 and sIL-6R concentrations, normal levels, levels in at-risk subjects on Day 1, and levels in ARDS subjects on Day 1 were extracted (median, 25th, 75th percentiles) and analyzed as log-normal distributions. $\mathrm{N}=300$ subjects were simulated from these lognormal distributions. The degree of variability reported in Park et.al. exceed $100 \%$ for both IL-6 and sIL-6R in the at-risk and ARDS population, so between-patient variability is an important consideration. The following table reports summaries of virtual subject values, as well as the calculated IL-6:sIL-6R complex using the formula [IL-6:sIL6R $]=[\mathrm{IL}-6]^{*}[\mathrm{sIL}-6 \mathrm{R}] \mathrm{Kd}-1$ and assuming the reported values in Park et.al. are free IL-6 and free sIL-6R (Table 1).

Notably the induction level of IL-6 and sIL-6R in ARDS relative to normal is 370-fold and 4.88-fold, respectively. Given a reported Kd value for sIL-6R to IL-6 of $5500 \mathrm{pM}$, binding is not favored and the free moiety forms predominate over the complex IL-6:sIL-6R (CR). The induced IL-6:sIL-6R complex level in ARDS relative to normal is 1810 -fold. While out-of-scope for this analysis, affinity for the IL-6:sIL-6R complex for gp130 is in the range of $10 \mathrm{pM}$, so the predicted increase in available IL-6:sIL-6R complex should be understood in that context.

\section{Binding Constant Selection}

A review of the literature provided estimates for binding constants for SIL:IL-6 complex and TCZ:IL-6R complex. These values represented data across clinical and nonclinical studies, and modeling from clinical and nonclinical species. Binding constants for use in our model were set at the median value encountered, or 15 pM for SIL:IL-6 (Kd_SC in Figure 2) and 1241 pM for TCZ:sIL-6R (Kd_TR in Figure 2).

\section{Binding Models}

Figure 2 
where: $\mathrm{S}$ and $\mathrm{T}$ are the free concentrations of SIL and TCZ in BALF, respectively; C and R are the free concentrations of IL-6 cytokine and sIL-6R, respectively; SC is the concentration of SIL:IL-6 complex; TR is the concentration of TCZ:sIL-6R; CR is the concentration of sIL-6R bound to IL-6. Kd_SC, Kd_TR and Kd_CR are the equilibrium dissocation binding constants for SIL:IL-6, TCZ:sIL-6R and IL-6:sIL-6R, respectively.

$\mathrm{CR}$ is the complex that (presumably) signals though the ubiquitously expressed gp130 receptor.

In stoichiometric form:

- $\mathrm{S}+\mathrm{C}<-\mathrm{Kd} \_\mathrm{SC}->\mathrm{SC}$ where $\mathrm{Kd}_{-} \mathrm{SC}=\mathrm{S}^{*} \mathrm{C} \mathrm{SC}-1$

- $\mathrm{T}+\mathrm{R}<-\mathrm{Kd}_{-} \mathrm{TR}->\mathrm{TR}$ where $\mathrm{Kd}_{-} \mathrm{TR}=\mathrm{T} * \mathrm{R}$ TR-1

- $\mathrm{C}+\mathrm{R}<-\mathrm{Kd}_{-} \mathrm{CR}->\mathrm{CR}$ where $\mathrm{Kd} \mathrm{C}_{-} \mathrm{CR}=\mathrm{C}^{*} \mathrm{R}$ CR-1

These reactions were implemented as a system of ordinary differential equations (ODEs). Initial conditions of IL-6 (C) and sIL-6R (R ) were given from the BALF concentration data in normal, pre-ARDS and ARDS subjects from the above table. IL-6:sIL-6R (CR ) was calculated, as above. Binding constants are given for SIL:IL-6 (SC) of 15 pM, IL-6:sIL-6R (CR ) of 5500 and TCZ:sIL-6R (TR ) of 1241.

Solving the resulting equilibrium equations may be possible, but these authors opted instead to simply simulate from the (dynamic) ordinary-differential equations out to steady-state. Off-rates were set $0.1 \mathrm{~s}-1$ for each reaction, so on-rates were derived kon=koff Kd-1. Although the off-rate is much more rapid than is typical for antibodies, the simulations were run out to steady-state so this assumption plays no role in the simulation results. Simulations at off-rate values of 0.01 and $0.01 \mathrm{~s}-1$ were performed to confirm similar results at equilibrium (time $>>$ koff-1.) Should an analytical solution to the equilibrium equations be derived, the results would be expected to match these.

The model solutions for C (IL-6), R (sIL-6R) and CR (IL-6:sIL-6R complex) at binding equilibrium were produced for each synthetic subject.

\section{Results}

The binding models were used to generate simulated BALF concentrations of IL-6, sIL-6R and the IL-6:sIL6R complex for normal subjects, subjects at risk of developing ARDS, and subjects with ARDS. Each model was used to simulate concentrations from 300 virtual subjects. Complex concentrations were simulated under four conditions: without treatment, treatment with TCZ, treatment with SIL, and treatment with TCZ + SIL. Results are displayed in Figure 3.

Concentrations simulated from the virtual group of subjects without ARDS and not at risk for ARDS are represented as Normal subjects. Dashed lines capture $90 \%$ of the simulated Normal Subject with Treatment: None cases. Observed concentrations of IL-6 and IL-6R [5] are summarized and plotted as Treatment: None.

Simulation of concentrations in at-risk subjects and subjects with ARDS following no treatment (black circles), treatment with TCZ (orange circles), SIL (blue circles) and TCZ + SIL (green circles) are summarized and plotted (point:median, interval: $5^{\text {th }}-95^{\text {th }}$ percentile for $\mathrm{n}=300$ simulated subjects.)

IL-6 and IL-6:sIL-6R are greatly elevated in both simulated populations, while sIL-6R elevations are more modest.

With TCZ intervention, IL-6 levels are unaffected and sIL-6R is reduced somewhat below the Normal case. IL-6:sIL-6R complex only slightly decreased relative to the no-intervention case. While this is somewhat counterintuitive, IL-6 competes with TCZ for sIL-6R and IL-6 is greatly induced in at-risk (corresponding to a WHO Score of 3-4 [36]) and ARDS populations (corresponding to a WHO Score of 5-7 [36]). This idea is consistent with the findings of Swaroopa et al. [3], where APACHE II score, together with Day 1 serum IL-6 and serum-IL-8 concentrations predicted survival in ARDS patients. 
With SIL intervention, sIL-6R levels are unaffected and IL-6 is greatly reduced below the Normal case. IL-6:sIL-6R complex is greatly decreased relative to the no-intervention case. Here, sIL-6R competes with SIL for IL- 6 and sIL-6R is only modestly induced in at-risk and ARDS populations.

With TCZ+SIL intervention, IL-6 and sIL-6R levels are reduced below the Normal case and achieve suppression equivalent to monotherapy results for their respective targets. Interestingly, IL-6:sIL-6R complex reduction is predicted to be greater than monotherapy. This reflects sequestration of both components of the complex and the nonlinear binding equilibrium.

\section{Discussion}

Cytokine signaling through the IL-6 pathway is complex and depends on multiple factors, including cell type and agonist or antagonist concentration in the tissue environment.

Though IL-6R is necessary for IL-6 signaling, and is a reasonable target for IL-6 signaling inhibition, the increase in IL-6 in ARDS is 30-50 times greater than the increase in IL-6R. Near-complete receptor occupancy of IL- 6 by TCZ in the lung could be necessary to achieve a robust decrease in signal transduction. An ability to achieve this depends on three factors: the relative concentrations of IL- 6 and sIL-6; the concentrations of antibodies such as SIL and TCZ; and the strength of the binding in each of their respective complexes (SIL:IL-6, TCZ:sIL-6R, and sIL-6R:IL-6).

Observational data on IL-6 and sIL-6R induction in ARDS suggests IL-6 is greatly upregulated, with concentrations reaching 370 -fold normal levels, while sIL-6R is only modestly increased, with concentrations reaching 4.88-fold normal levels. Maximal achievable serum siltuxumab concentration exceeds TCZ concentration by approximately two-fold. The dissocation equilibirum concentration for SIL is two to three orders-of-magnitude lower than TCZ, meaning that SIL is better at sequestering IL-6 than TCZ is at sequestering sIL-6. Taking these three factors into account, results of the simulation greatly favor the use of SIL over TCZ to inhibit IL-6 signaling. However, these results are dependent on several key assumptions:

The value for antibody penetration (BALF:serum ratio) of SIL or TCZ has not been reported. The value used in the simulation $(0.2 \%)$ represented the upper-end of reported values for unrelated monoclonal antibodies. Clearly, having experimentally-obtained penetration values for these compounds would be ideal, but difficult to source for repurposed drugs where the lung has not been a studied as a site of action.

The binding model introduced here is complex, yet only captures a portion of the interactions involved in IL-6 signaling. Specifically, IL-6 binds to both sIL-6R (via the trans pathway in all cells) and membranebound IL-6R (mIL-6R) (via the classic pathway on certain cells such as some immune cells). mIL-6R, and therefore classic signaling, are not included in the model. Similarly, soluble gp130 (sgp130) (an important IL-6 modulator in the trans pathway) was not included in the model. Signaling complex formation (IL6:sIL-6R:gp130 and IL-6:mIL-6R:gp130) is not accounted for directly in the model.

Still, suppression of free IL-6 reduces IL-6:mIL-6R and IL-6:sIL-6R, and IL-6:sIL-6R is tracked in the model. If anything, the omission of mIL-6R from the model underscores the relative importance of IL- 6 suppression relative to sIL-6R suppression. While signaling complex formation is not tracked in the model (for classic or trans signaling), gp130 is constitutively expressed and is therefore assumed not to be a limiting factor in IL-6 signaling. Similarly, gp130 transmembrane protein binding is not included in the model. However, gp130 affinity for IL-6:sIL-6R is higher than IL-6 for sIL-6R, suggesting that the limiting step is the binding of IL-6 to sIL-6R.

Finally, a review of the literature revealed a range of binding constants reported for SIL:IL-6 and TCZ:sIL-6R (Table 2). The selection of Kd for use in the model was the median reported value (Table 2, Figure 2). The impact of the true Kds being lower or higher is not accounted for in this work. 


\section{Considerations for Place in Therapy}

A rapid, coordinated innate immune response is the initial line of defense against viral infections. Hyperinflammatory responses, however, can cause immunopathology. Low pathogenic coronaviruses typically infect the upper airways, while highly pathogenic coronaviruses infect the lower respiratory tract and can cause severe pneumonia, sometimes leading to acute lung injury and ARDS. Disease severity of the highly pathogenic coronaviruses SARS and MERS was influenced by factors such as initial viral titers in the airways, age, and comorbid conditions [28].

The clinical course of SARS progressed in three stages. Robust viral replication dominated the first phase, which lasted a few days. The second phase was associated with high fever, hypoxemia and progression to pneumonia despite a decrease in viral load. The third phase is characterized by strong inflammatory response, in which $20 \%$ of patients progressed to ARDS and often death [29]. MERS progresses more rapidly, and has a higher fatality rate than SARS. Common clinical manifestations of MERS resemble those of SARS-CoV-2 and include rapid progression to pneumonia [30, 31]. Like SARS-CoV-2, the majority of MERS patients with shortness of breath progressed to severe pneumonia and required admission to the ICU. Analyses of lungs from patients who died from SARS-CoV showed infection of both the airway and alveolar epithelial cells, vascular endothelial cells, macrophages, monocytes and lymphocytes. Neutrophils and macrophages extensively infiltrated cells [32]. The only tissue samples available for MERS is the analysis of lung tissue from one patient, which were consistent with what was seen in SARS [33].

Virus-induced cytopathic effects and viral evasion of host immune responses are thought to play major roles in disease severity. This argues for antiviral therapy as early as possible in treatment, with adjunctive immunotherapy during the time when patients are at risk for ARDS and in early ARDS. This paradigm would be similar to therapeutic interventions aimed at MERS viral load reduction, which were somewhat beneficial when administered early (but not later in) MERS-CoV infection [30, 34, 35].

IL-6 concentrations skyrocket at the onset of ARDS (i.e. WHO Score [?] 5 [36]). Immediate treatment with an antibody such as SIL upon hospital admission for patients with low oxygenation needs (i.e. $200 \mathrm{mmHg}$ $<\mathrm{PaO} 2$ to FIO2 [?]300 $\mathrm{mmHg}$ with positive end-expiratory pressure or continuous positive airway pressure [?] $5 \mathrm{cmH} 2 \mathrm{O}$, delivered invasively or noninvasively; Corresponding to WHO Score of 4 [36]) could drive the immune response out of hyperreactivity. SIL treatment in early ARDS (i.e. Days 1-3) would be crucial to preventing the IL-6 onslaught that leads to lung damage.

Additionally, treatment with an antibody such as TCZ in patients at risk of developing ARDS (i.e. WHO Score of $3[36]$ ) could mediate the IL-6 signaling response synergistically. Preliminary data suggest IL$6 \mathrm{R}$ blockade is most effective in critically ill patients, presumably because ARDS has progressed and IL-6 concentrations have already returned to near-normal. For example, Regeneron and Sanofi are amending their phase 3 trial evaluating sarilumab, an IL-6R antagonist, in hospitalized patients with "severe" or "critical" illness caused by COVID-19 to include only "critical" patients, based on preliminary results that sarilumab provided a clinical benefit to patients requiring mechanical ventilation or high-flow oxygenation or treatment in an intensive care unit, but no notable benefit on clinical outcomes in fpatients who required oxygen supplementation without mechanical or high-flow oxygenation [37].

\section{Conclusions}

Antibody penetration into the lung has been studied for anti-RSV antibodies. There, BALF:serum concentration ratios are reported in the range $0.1-0.2 \%$. While it is tempting to make dose selection evaluations based on systemic exposure alone, solely relying on this information would greatly overestimate target binding in lung BALF. Binding equilibrium is related to concentrations of each species and binding constants, so understanding the concentration of each species at the site of action is crucial.

An important caveat of these simulations is to understand that they are made 1) based on the assumption 
that the maximal drug concentration instantaneously arrives in the BALF at a pre-defined ratio and 2) equilibrium binding occurs instantaneously and total target concentrations do not change over time. We would expect that unbound TCZ or SIL would constantly cycle into the BALF allowing for more sIL-6R or IL-6, respectively, to be bound. However, TCZ or SIL concentrations would also be falling relative to the maximal concentrations used here. Moreover, these simulations do not account for the synthesis and turn-over rates of sIL-6R and IL-6. If IL-6 is formed and turned over more rapidly, that would tilt the results towards TCZ's favor. Far more complex, dynamic calculations would be required to understand the push-pull of these effects on binding outcomes. Additionally, variability in TCZ and SIL pharmacokinetics should be considered in more complex simulations.

We are unaware of specific BALF data from COVID-19 subjects and are relying on the translatability of general ARDS cases to the COVID-19 context. Clearly, more specific data from this patient type would be of greater utility and specificity. Moreover, the BALF concentration pattern changes over time, suggesting longitudinal data would provide insight on the timing of treatment.

Care should be taken to not over-interpret these simulations to suggest that SIL should be preferred over TCZ. To the contrary, these simulations suggest that the combined effect of these drugs is better than the monotherapy results. In the context of so much uncertainty around target concentrations, target dynamics and relative importance of each target's contributions, combination therapy should be considered. Finally, it should be noted that anti-IL-6 therapy, anti-IL-6R therapy, and combination anti-IL-6:IL-6R therapy could have differing efficacies. The nuances of IL-6 signaling must be taken into account when discussing antibodies that target the IL-6 signaling pathway, and we must not treat IL- 6 and IL-6R targets or blockers interchangeably.

\section{Acknowledgments}

The authors would like to acknowledge Synchrogenix for medical writing assistance.

\section{Conflict of Interest Statement}

All authors are employees of Certara, Inc.

\section{Funding Information}

Funding for this work was made possible in part by a grant from the Bill and Melinda Gates Foundation.

\section{Data Availability Statement}

Data for model development to support the findings of this study were derived from public domain resources:

\begin{tabular}{lll}
\hline Data & Reference & Reference \\
\hline TCZ:sIL-6R Kd & PMID 22101760 & DOI 10.1007/s10928-011-9227-z \\
SIL:IL-6 Kd & PMID 15239138 & DOI 10.1002/ijc.20270 \\
TCZ Cmax & PMID 29622697 & DOI \\
& & $10.1634 /$ theoncologist.2018-0028 \\
SIL Cmax & Sylvant (SILTUXIMAB) BLA & Sylvant (SILTUXIMAB) BLA \\
& $\# 125496$ Drugs@FDA & $\# 125496$ Drugs@FDA \\
\hline
\end{tabular}

\section{References}

[1] . 2000;342:1334-1349.

[2] Fanelli et al. Acute respiratory distress syndrome: new definition, current and future therapeutic options. $J$ Thorac Dis 2013;5(3):326-334. 
[3] Swaroopa et al. Association of serum interleukin-6, interleukin-8, and Acute Physiology and Chronic Health Evaluation II score with clinical outcome in patients with acute respiratory distress syndrome. Indian J Crit Care Med. 2016; 20(9):518-525.

[4] Didion SP. Cellular and oxidative mechanisms associated with interleukin-6 signaling in the vasculature. Int J Mol Sci. 2017; 18, 2563.

[5] Park WY, Goodman RB, Steinberg KP. Cytokine balance in the lungs of patients with acute respiratory distress syndrome. Am J Respir Crit Care Med. 2001;164:1896-1903.

[6] Meduri GU, Headley S, Kohler G, et al. Peristent elevation of inflammatory cytokines predicts a poor outcome in ARDS. Plasma IL-1 beta and IL-6 levels are consistent and efficient predictors of outcome over time. Chest. 1995;107:1062-1073.

[7] Ding C, Jones G. Technology evaluation: MRA, Chugai. Current Opinion in Molecular Therapeutics. 2003; 5(1):64-69.

[8] Ito H. IL-6 and Crohn's Disease. Curr Drug Targets Inflamm Allergy. 2003; 2(2):125-30.

[9] Neelapu SS, Tummala S, Kebriaei P, et al. Chimeric antigen receptor T cell therapy-assessment and management of toxicities. Nat Rev Clin Oncol. 2018;15(1):47.

[10] Khadka, Sakemura, Kenderian, Johnson. Management of cytokine release syndrome: an update on emerging antigen-specific T cell engaging immunotherapies. Immunotherapy. 2019;11(10):851-857.

[11] Cross LJM, Matthay MA. Biomarkers in acute lung injury: insights into pathogenesis of acute lung injury. Crit Care Clin. 2011 April;27(2):355-377.

[12] Parsons PE, Eisner MD, Thompson BT, et al. Lower tidal volume ventilation and plasma cytokine markers of inflammation in patients with acute lung injury. Crit Care Med. 2005;33:1-6.

[13] Scheller J, Chalaris A, Schmidt-Arras D, Rose-John S. The Pro- And Anti-Inflammatory Properties of the Cytokine interleukin-6. Biochim Piophys Acta. 2011; 1813(5): 878-88.

[14] Chalaris A, Rabe B, Paliga K, et al. Apoptosis is a natural stimulus of IL6R shedding and contributes to the pro-inflammatory trans-signaling function of neutrophils. Blood. 2007;110:1748-1755.

[15] Rabe B, Chalaris A, May U, et al. Transgenic blockade of interleukin 6 transsignaling abrogates inflammation. Blood. 2008;111:1021-1028.

[16] Becker C, Fantini MC, Schramm C, et al. TGF-beta suppresses tumor progression in colon cancer by inhibition of IL-6 trans-signaling. Immunity. 2004;21:491-501.

[17] Chen Q, Fisher DT, Clancy KA, et al. Fever-range thermal stress promotes lymphocyte trafficking across high endothelial venules via an interleukin 6 trans-signaling mechanism. Nat Immunol. 2006;7:1299-1308.

[18] Oberg HH, Wesch D, Grussel S, Rose-John S, Kabelitz D. Differential expression of CD126 and CD130 mediates different STAT-3 phosphorylation in CD4+CD25- and CD25 high regulatory T cells. Int Immunol. 2006; $18: 555-563$.

[19] Rose-John S, Waetzig GH, Scheller J, Grotzinger J, Seegert D. The IL-6/sIL-6R complex as a novel target for therapeutic approaches., Expert Opin Ther Targets. 2007;11;613-624.

[20] Scheller J, Rose-John S. Interleukin-6 and its receptor: from bench to bedside. Med Microbiol Immunol. 2006;195:173-183.

[21] Jostock T, Müllberg J, Özbek S, et al. Soluble gp130 is the natural inhibitor of soluble IL-6R transsignaling responses. Eur J Biochem. 2001;268:160-167.

[22] Rose-John. IL-6 trans-signaling via the Soluble il-6 receptor: importance for the pro-inflammatory activities of IL-6. Int J Biol Sci. 2012;8(9):1237-1247. 
[23] Sylvant Highlights of Prescribing Information. https://www.accessdata.fda.gov/drugsatfda_docs/label/2014/125496s000lbl.pdf

[24] . Int. J. Cancer. 2004; 111: 592-595.

[25] Le RQ et al., FDA Approval Summary: Tocilizumab for Treatment of Chimeric Antigen Receptor T Cell-Induced Severe or Life-Threatening Cytokine Release Syndrome. The Oncologist 2018; 23:943-947.

[26] Gibiansky and Frey. Linking interleukin-6 receptor blockade with tocilizumab and its hematological effects using a modeling approach. J Pharmacokinet Pharmacodyn. 2012; 39:5-16.

[27] Tabrizi M, Bornstein GG, Suria H. Biodistribution mechanisms of therapeutic monoclonal antibodies in health and disease. The AAPS Journal. 2010; 12(1): 33-43.

[28] Channappanavar and Perlman. Pathogenic human coronavirus infections: causes and consequences of cytokine storm and immunopathology. Semin Immunopathol. 2017; 39:529-539.

[29] Peiris JS et al. Clinical progression and viral load in a community outbreak of coronavirus-associated SARS pneumonia: a prospective study. Lancet 2003; 361(9371):1767-1772.

[30] Al-Tawfiq JA, et al. Middle East respiratory syndrome coronavirus: a case-control study of hospitalized patients. Clin Infect Dis. 2014;59(2):160-165.

[31] Arabi YM, et al. Clinical course and outcomes of critically ill patients with Middle East respiratory syndrome coronavirus infection. Ann Intern Med. 2014;160:389-397.

[32] Gu J, et al. Multiple organ infection and the pathogenesis of SARS. J Exp Med. 2005;202(3):415-424.

[33] Ng DL, et al. Clinicopathologic, immunohistochemical, and ultrastructural findings of a fatal case of Middle East respiratory syndrome coronavirus infection in the United Arab Emirates, April 2014. Am J Pathol. 2016;186(3):652-658.

[34] Falzarano D, et al. Treatment with interferon-alpha2b and ribavirin improves outcome in MERS-CoVinfected rhesus macaques. Nat Med. 2013;19(10):1313-1317.

[35] Omrani AS, et al. Ribavirin and interferon alfa-2a for severe Middle East respiratory syndrome coronavirus infection: a retrospective cohort study. Lancet Infect Dis. 2014;14(11):1090-1095.

[36] WHO R\&D Blueprint: Covid 19: Informal consultation on the potential role of IL-6/IL-1 antagonists in the clinical management of COVID 19 infection.

[37] Regeneron and Sanofi Provide Update on US Phase 2/3 Adaptive-Designed Trial of Kevzaraß) (Sarilumab) in Hospitalized COVID-19 Patients. News Release. Regeneron; April 27, 2020. Accessed April 27, 2020. https://investor.regeneron.com/news-releases/news-release-details/regeneron-and-sanofi-provideupdate-us-phase-23-adaptive.

[38] van Zaanen HC, Koopmans RP, Aarden LA, Rensink HJ, Stouthard JM, Warnaar SO, Lokhorst HM, van Oers MH.J Clin Invest. Endogenous interleukin 6 production in multiple myeloma patients treated with chimeric monoclonal anti-IL6 antibodies indicates the existence of a positive feed-back loop.1996 Sep $15 ; 98(6): 1441-8$.

[39] Deisseroth A, Ko CW, Nie L, Zirkelbach JF, Zhao L, Bullock J, Mehrotra N, Del Valle P, Saber H, Sheth C, Gehrke B, Justice R, Farrell A, Pazdur R. FDA Approval: Siltuximab for the Treatment of Patients with Multicentric Castleman Disease. Clin Cancer Res. 2015 Mar 1;21(5):950-4.

[40] Wang, W., Wang, X., Doddareddy, R. et al. Mechanistic Pharmacokinetic/Target Engagement/Pharmacodynamic (PK/TE/PD) Modeling in Deciphering Interplay Between a Monoclonal Antibody and Its Soluble Target in Cynomolgus Monkeys. AAPS J 2014; 16, 129-139. 
[41] Mihara M, Kasutani K, Okazaki M, Nakamura A, Sawai S, Sugimoto M, Matsumoto Y, Ohsugi Y. Tocilizumab inhibits signal transduction mediated by both mIL-6R and sIL-6R, but not by the receptors of other members of IL-6 cytokine family. International Immunopharmacology 2005; 5(12): 1731-1740.

Figure 1: IL-6 Classic and Trans Signaling Pathways

(a) Classic Pathway

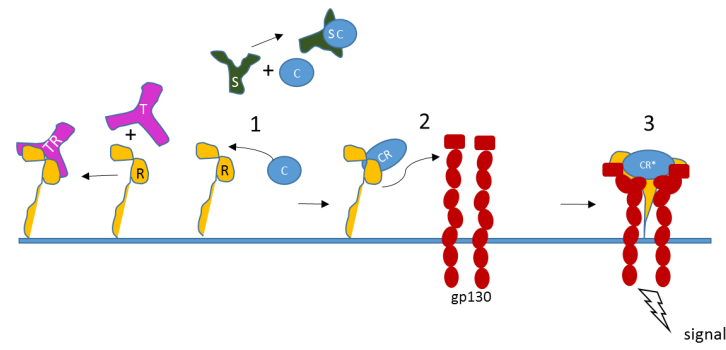

C: IL-6; S: siltuximab; SC: IL-6:siltuximab complex; R: IL-6R; T: TCZ; TR: IL-6R:TCZ complex; CR: IL-6:IL-6R pre-signaling complex; CR*: IL-6:IL-6R complex bound to gp130 (signaling complex)

(b) Trans Pathway

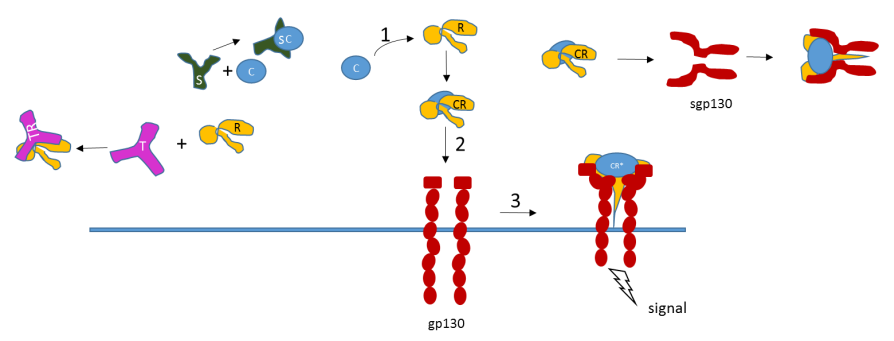

C: IL-6; S: siltuximab; SC: IL-6:siltuximab complex; R: sIL-6R; T: tocilizumab; TR: sIL6R:TCZ complex; CR: IL-6:sIL-6R pre-signaling complex; CR*: IL-6:sIL-6R complex bound to gp130 (signaling complex) 


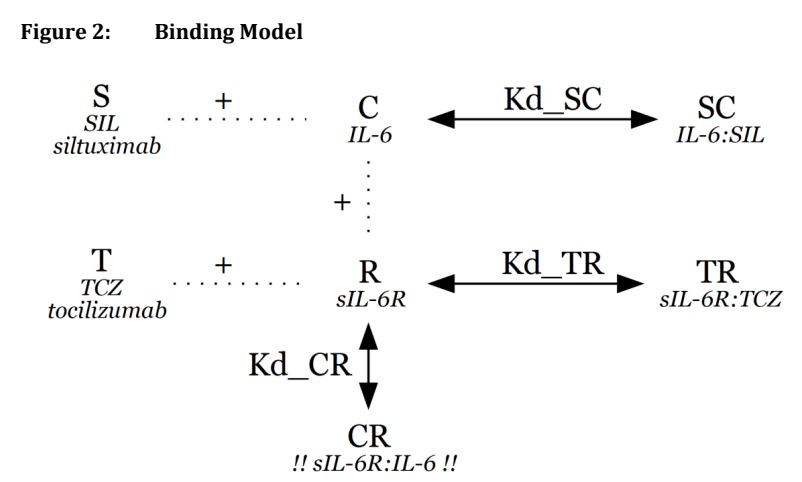

C: IL-6; S: siltuximab; SC: IL-6:siltuximab complex; R: sIL-6R; T: tocilizumab; TR: sIL6R:tocilizumab complex; CR: IL-6:sIL-6R pre-signaling complex; Kd: binding constant 


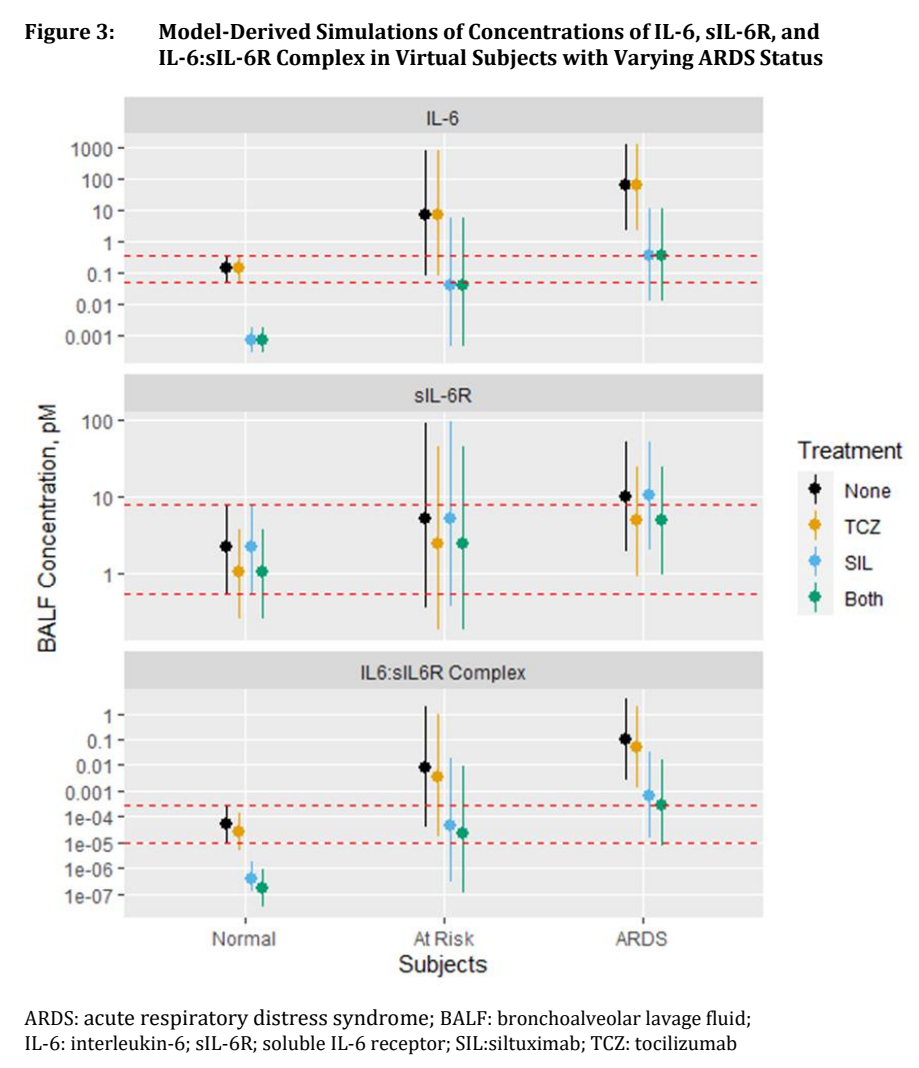

\section{Hosted file}

BJCP Table 1.docx available at https://authorea.com/users/332276/articles/458718-covid-19simulation-study-of-tocilizumab-and-siltuximab-interventions-in-the-context-of-acuterespiratory-distress-syndrome

\section{Hosted file}

BJCP Table 2.docx available at https://authorea.com/users/332276/articles/458718-covid-19simulation-study-of-tocilizumab-and-siltuximab-interventions-in-the-context-of-acuterespiratory-distress-syndrome 